\title{
Genotypic variability enhances the reproducibility of an ecological study
}

Article

Accepted Version

Milcu, A., Puga-Freitas, R., Ellison, A. M., Blouin, M., Scheu, S., Freschet, G. T., Rose, L., Barot, S., Cesarz, S., Eisenhauer, N., Girin, T., Assandri, D., Bonkowski, M., Buchmann, N., Butenschoen, O., Devidal, S., Gleoxner, G., Gessler, A., Gigon, A., Greiner, A., Grignani, C., Hansart, A., Kayler, Z., Lange, M., Lata, J. C., Le Galliard, J. F., Lukac, M. ORCID: https://orcid.org/0000-0002-8535-6334, Mannerheim, N., Muller, M. E. H., Pando, A., Rotter, P., Scherer-Lorenzen, M., Seyhun, R., Urban-Maed, K., Weigelt, A., Zavattaro, L. and Roy, J. (2018) Genotypic variability enhances the reproducibility of an ecological study. Nature Ecology \& Evolution, 2 (2). pp. 279-287. ISSN 2397-334X doi: https://doi.org/10.1038/s41559-017-0434-x Available at https://centaur.reading.ac.uk/74258/

It is advisable to refer to the publisher's version if you intend to cite from the work. See Guidance on citing.

To link to this article DOI: http://dx.doi.org/10.1038/s41559-017-0434-x

Publisher: Nature 
including copyright law. Copyright and IPR is retained by the creators or other copyright holders. Terms and conditions for use of this material are defined in the End User Agreement.

\section{www.reading.ac.uk/centaur}

\section{CentAUR}

Central Archive at the University of Reading

Reading's research outputs online 


\section{Genotypic variability enhances the reproducibility of an ecological study}

2 Alexandru Milcu ${ }^{1,2}$, Ruben Puga-Freitas ${ }^{3}$, Aaron M. Ellison ${ }^{4,5}$, Manuel Blouin ${ }^{3,6}$, Stefan Scheu ${ }^{7}$,

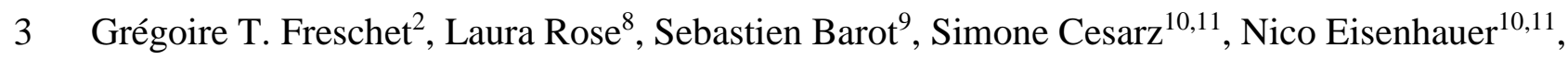

4 Thomas Girin $^{12}$, Davide Assandri ${ }^{13}$, Michael Bonkowski ${ }^{14}$, Nina Buchmann ${ }^{15}$, Olaf

5 Butenschoen $^{7,16}$, Sebastien Devidal ${ }^{1}$, Gerd Gleixner ${ }^{17}$, Arthur Gessler ${ }^{18,19}$, Agnès Gigon ${ }^{3}$, Anna

6 Greiner $^{8}$, Carlo Grignani ${ }^{13}$, Amandine Hansart ${ }^{20}$, Zachary Kayler ${ }^{19,21}$, Markus Lange ${ }^{17}$, Jean-

7 Christophe Lata ${ }^{22}$, Jean-François Le Galliard ${ }^{20,22}$, Martin Lukac ${ }^{23,24}$, Neringa Mannerheim ${ }^{15}$,

8 Marina E.H. Müller ${ }^{18}$, Anne Pando ${ }^{6}$, Paula Rotter $^{8}$, Michael Scherer-Lorenzen ${ }^{8}$, Rahme

9 Seyhun $^{22}$, Katherine Urban-Mead ${ }^{2}$, Alexandra Weigelt ${ }^{10,11}$, Laura Zavattaro ${ }^{13}$ and Jacques Roy ${ }^{1}$

$10{ }^{1}$ Ecotron (UPS-3248), CNRS, Campus Baillarguet, F-34980, Montferrier-sur-Lez, France.

$11{ }^{2}$ Centre d'Ecologie Fonctionnelle et Evolutive, CEFE-CNRS, UMR 5175, Université de

12 Montpellier - Université Paul Valéry - EPHE, 1919 route de Mende, F-34293, Montpellier

13 Cedex 5, France.

$14{ }^{3}$ Institut des Sciences de l'Ecologie et de l'Environnement de Paris (UPMC, UPEC, Paris Diderot,

15 CNRS, IRD, INRA), Université Paris-Est Créteil, 61 avenue du Général De Gaulle, F-94010

16 Créteil Cedex, France.

$17{ }^{4}$ Harvard Forest, Harvard University, 324 North Main Street, Petersham, Massachusetts, USA.

$18{ }^{5}$ University of the Sunshine Coast, Tropical Forests and People Research Centre, Locked Bag 4,

19 Maroochydore DC, Queensland 4558, Australia.

$20{ }^{6}$ Agroécologie, AgroSup Dijon, INRA, Univ. Bourgogne Franche-Comté, F-21000 Dijon, France

$21{ }^{7}$ J.F. Blumenbach Institute for Zoology and Anthropology, Georg August University Göttingen,

22 Berliner Str. 28, 37073 Göttingen, Germany.

$23{ }^{8}$ Faculty of Biology, University of Freiburg, Geobotany, Schaenzlestr. 1, D-79104 Freiburg,

24 Germany.

$25{ }^{9}$ IRD, Institut des Sciences de l'Ecologie et de l'Environnement de Paris (UPMC, UPEC, Paris

26 Diderot, CNRS, IRD, INRA), UPMC, Bâtiment 44-45, deuxième étage, bureau 208, CC 237, 4

27 place Jussieu, 75252 Paris cedex 05, France. 
$28{ }^{10}$ German Centre for Integrative Biodiversity Research (iDiv), Halle-Jena-Leipzig, Deutscher 29 Platz 5e, 04103 Leipzig, Germany.

$30{ }^{11}$ Institute of Biology, Leipzig University, Deutscher Platz 5e, 04103 Leipzig, Germany.

$31{ }^{12}$ Institut Jean-Pierre Bourgin, INRA, AgroParisTech, CNRS, Université Paris-Saclay, RD10,

3278026 Versailles Cedex, France.

$33{ }^{13}$ Department of Agricultural, Forest and Food Sciences, University of Turin, largo Braccini, 2,

3410095 Grugliasco, Italy.

$35{ }^{14}$ Cluster of Excellence on Plant Sciences (CEPLAS), Terrestrial Ecology Group, Institute for

36 Zoology, University of Cologne, Zülpicher Str. 47b, 50674 Köln, Germany.

$37{ }^{15}$ Institute of Agricultural Sciences, ETH Zurich, Universitätsstrasse 2, 8092 Zürich, Switzerland

$38{ }^{16}$ Senckenberg Biodiversität und Klima Forschungszentrum BiK-F, Georg-Voigt-Straße 14-16,

39 Frankfurt am Main.

$40 \quad{ }^{17}$ Max Planck Institute for Biogeochemistry, Postfach 100164, 07701 Jena, Germany.

$41{ }^{18}$ Leibniz Centre for Agricultural Landscape Research (ZALF), Institute of Landscape

42 Biogeochemistry, Eberswalder Str. 84, 15374 Müncheberg, Germany.

$43{ }^{19}$ Swiss Federal Research Institute WSL, Zürcherstr. 111, 8903 Birmensdorf, Switzerland.

$44{ }^{20}$ Ecole normale supérieure, PSL Research University, Département de biologie, CNRS, UMS

45 3194, Centre de recherche en écologie expérimentale et prédictive (CEREEP-Ecotron

46 IleDeFrance), 78 rue du château, 77140 Saint-Pierre-lès-Nemours, France.

$47{ }^{21}$ Department of Soil and Water Systems, University of Idaho, 875 Perimeter Dr., Moscow, ID, 48 USA.

$49{ }^{22}$ Institut des Sciences de l'Ecologie et de l'Environnement de Paris (UPMC, UPEC, Paris

50 Diderot, CNRS, IRD, INRA), Sorbonne Universités, CC 237, 4 place Jussieu, 75252 Paris cedex 5105 , France.

$52{ }^{23}$ School of Agriculture, Policy and Development, University of Reading, Reading, RG6 6AR, 53 UK.

$54 \quad{ }^{24}$ FLD, Czech University of Life Sciences, 16500 Prague, Czech Republic.

56 Corresponding author: Alexandru Milcu, CNRS, Ecotron - UPS 3248, Campus Baillarguet, 34980,

57 Montferrier-sur-Lez, France, email: alex.milcu@cnrs.fr, phone: +33 (0) 434-359-893. 
58 Many scientific disciplines are currently experiencing a "reproducibility crisis" because

59 numerous scientific findings cannot be repeated consistently. A novel but controversial

60 hypothesis postulates that stringent levels of environmental and biotic standardization in

61 experimental studies reduces reproducibility by amplifying impacts of lab-specific

62 environmental factors not accounted for in study designs. A corollary to this hypothesis is

63 that a deliberate introduction of controlled systematic variability (CSV) in experimental

64 designs may lead to increased reproducibility. We tested this hypothesis using a multi-

65 laboratory microcosm study in which the same ecological experiment was repeated in $\mathbf{1 4}$

66 laboratories across Europe. Each laboratory introduced environmental and genotypic CSV

67 within and among replicated microcosms established in either growth chambers (with

68 stringent control of environmental conditions) or glasshouses (with more variable

69 environmental conditions). The introduction of genotypic CSV led to lower among-

70 laboratory variability in growth chambers, indicating increased reproducibility, but had no

71 significant effect in glasshouses where reproducibility was generally lower. Environmental

72 CSV had little effect on reproducibility. Although there are multiple causes for the

73 "reproducibility crisis", deliberately including genetic variation may be a simple solution

74 for increasing the reproducibility of ecological studies performed in controlled

75 environments. Reproducibility — the ability to duplicate a study and its findings—is a defining feature of

78 scientific research. In ecology, it is often argued that it is virtually impossible to accurately

79 duplicate any single ecological experiment or observational study. The rationale is that the

80 complex ecological interactions between the ever-changing environment and the extraordinary 
81 diversity of biological systems exhibiting a wide range of plastic responses at different levels of

82 biological organization make exact duplication unfeasible ${ }^{1,2}$. Although this may be true for

83 observational and field studies, numerous ecological (and agronomic) studies are carried out with

84 artificially assembled simplified ecosystems and controlled environmental conditions in

85 experimental microcosms or mesocosms (henceforth, "microcosms") ${ }^{3-5}$. Since biotic and

86 environmental parameters can be tightly controlled in microcosms, results from such studies

87 should be easier to reproduce. Even though microcosms have frequently been used to address

88 fundamental ecological questions ${ }^{4,6,7}$, there has been no quantitative assessment of the

89 reproducibility of any microcosm experiment.

90 Experimental standardization - the implementation of strictly defined and controlled

91 properties of organisms and their environment—is widely thought to increase both

92 reproducibility and sensitivity of statistical tests ${ }^{8,9}$ because it reduces within-treatment

93 variability. This paradigm has been recently challenged by several studies on animal behavior,

94 suggesting that stringent standardization may, counterintuitively, be responsible for generating

95 non-reproducible results ${ }^{9-11}$ and contribute to the actual reproducibility crisis ${ }^{12-15}$; the results

96 may be valid under given conditions (i.e., they are local "truths") but are not generalizable ${ }^{8,16}$.

97 Despite rigorous adherence to experimental protocols, laboratories inherently vary in many

98 conditions that are not measured and are thus unaccounted for, such as experimenter, micro-scale

99 environmental heterogeneity, physico-chemical properties of reagents and lab-ware, pre-

100 experimental conditioning of organisms, and their genetic and epigenetic background. It even has

101 been suggested that attempts to stringently control all sources of biological and environmental

102 variation might inadvertently lead to the amplification of the effects of these unmeasured

103 variations among laboratories, thus reducing reproducibility ${ }^{9-11}$. 
Some studies have gone even further, hypothesizing that the introduction of controlled

105 systematic variation (CSV) among the replicates of a treatment (e.g., using different genotypes or

106 varying the organisms' pre-experimental conditions among the experimental replicates) should

107 lead to less variable mean response values between the laboratories that duplicate the

108 experiments ${ }^{9,11}$. In short, it has been argued that reproducibility may be improved by shifting the

109 variance from among experiments to within them ${ }^{9}$. If true, then introducing CSV will increase

110 researchers' ability to draw generalizable conclusions about the directions and effect sizes of

111 experimental treatments and reduce the probability of false positives. The trade-off inherent to

112 this approach is that increasing within-experiment variability will reduce the sensitivity (i.e. the

113 probability of detecting true positives) of statistical tests. However, it currently remains unclear

114 whether introducing CSV increases reproducibility of ecological microcosm experiments, and if

115 so, at what cost for the sensitivity of statistical tests.

116 To test the hypothesis that introducing CSV enhances reproducibility in an ecological

117 context, we had 14 European laboratories simultaneously run a simple microcosm experiment

118 using grass (Brachypodium distachyon L.) monocultures and grass and legume (Medicago

119 truncatula Gaertn.) mixtures. As part of the reproducibility experiment, the 14 laboratories

120 independently tested the hypothesis that the presence of the legume species $M$. truncatula in

121 mixtures would lead to higher total plant productivity in the microcosms and enhanced growth of

122 the non-legume B. distachyon via rhizobia-mediated nitrogen fertilization and/or nitrogen

123 sparing effects ${ }^{17-19}$.

124 All laboratories were provided with the same experimental protocol, seed stock from the

125 same batch, and identical containers in which to establish microcosms with grass only and grass-

126 legume mixtures. Alongside a control (CTR) with no CSV and containing a homogenized soil 
127 substrate (mixture of soil and sand) and a single genotype of each plant species, we explored the

128 effects of five different types of within- and among-microcosm CSV on experimental

129 reproducibility of the legume effect (Fig. 1): 1) within-microcosm environmental CSV (ENVW)

130 achieved by spatially varying soil resource distribution through the introduction of six sand

131 patches into the soil; 2) among-microcosm environmental CSV $\left(\mathrm{ENV}_{\mathrm{A}}\right)$, which varied the

132 number of sand patches (none, three, or six) among replicate microcosms; 3) within-microcosm

133 genotypic CSV $\left(\mathrm{GEN}_{\mathrm{W}}\right)$ that used three distinct genotypes per species planted in homogenized

134 soil in each microcosm; 4) among-microcosm genotypic CSV (GEN $\left.\mathrm{A}_{\mathrm{A}}\right)$ that varied the number of

135 genotypes (one, two, or three) planted in homogenized soil among replicate microcosms; and 5)

136 both genotypic and environmental $\mathrm{CSV}\left(\mathrm{GEN}_{\mathrm{W}}+\mathrm{ENV}_{\mathrm{W}}\right)$ within microcosms that used six sand

137 patches and three plant genotypes per species in each microcosm. In addition, we tested whether

138 CSV effects are modified by the level of standardization within laboratories by using two

139 common experimental approaches ('SETUP' hereafter): growth chambers with tightly controlled

140 environmental conditions and identical soil (eight laboratories) or glasshouses with more loosely

141 controlled environmental conditions and different soils (six laboratories; see Supplementary

142 Table 1 for the physico-chemical properties of the soils).

143 We measured 12 parameters representing a typical ensemble of response variables reported

144 for plant-soil microcosm experiments. Six of these were measured at the microcosm-level: shoot

145 biomass, root biomass, total biomass, shoot-to-root ratio, evapotranspiration, and decomposition

146 of a common substrate using a simplified version of the "teabag litter decomposition method"20.

147 The other six were measured on B. distachyon alone: seed biomass, height, and four shoot-tissue

148 chemical variables; $\mathrm{N} \%, \mathrm{C} \%, \delta^{15} \mathrm{~N}, \delta^{13} \mathrm{C}$. All 12 variables were then used to calculate the effect

149 of the presence of a nitrogen-fixing legume on ecosystem functions in grass-legume mixtures 
150 ('net legume effect' hereafter) (Supplementary Table 2), calculated as the difference between the

151 values measured in the microcosms with and without legumes, an approach often used in

152 legume-grass binary cropping systems ${ }^{19,21}$ and biodiversity-ecosystem function experiments ${ }^{17,22}$.

153 Statistically significant differences among the 14 laboratories were considered an indication

154 of irreproducibility. In the first instance, we assessed how our experimental treatments (CSV and

155 SETUP) affected the number of laboratories that produced results that could be considered to

156 have reproduced the same finding. We then determined how experimental treatments affected

157 standard deviation (SD) of the legume effect for each of the 12 variables both within- and

158 among-laboratories; lower among-laboratory SD implies that the results were more similar,

159 suggesting increased reproducibility. Lastly, we explored the relationship between within- and

160 among-laboratory SD, and how the experimental treatments affected the statistical power of

161 detecting the net legume effect.

162 Although each laboratory followed the same experimental protocol, we found a remarkably

163 high level of among-laboratory variation for most response variables (Supplementary Fig. 1) and

164 the net legume effect on those variables (Fig. 2). For example, the net legume effect on mean

165 total plant biomass varied among laboratories from 1.31 to $6.72 \mathrm{~g}$ dry weight (DW) per

166 microcosm in growth chambers, suggesting that unmeasured laboratory-specific conditions

167 outweighed effects of experimental standardization. Among glasshouses, differences were even

168 larger: the net legume effect on mean plant biomass varied by two orders of magnitude, from

1690.14 to $14.57 \mathrm{~g}$ DW per microcosm (Fig. 2). Furthermore, for half of the variables (root biomass,

170 litter decomposition, grass height, foliar $\mathrm{C} \%, \delta^{15} \mathrm{C}$ and $\delta^{15} \mathrm{~N}$ ) the direction of the net legume

171 effect varied with laboratory. 
Mixed-effects models were used to test the effect of legume species presence (LEG),

173 laboratory (LAB), CSV, and their interactions (with experimental block-within-LAB growth

174 chamber or glasshouse bench — as a random factor) on the 12 response variables. The impact of

175 the presence of legumes varied significantly with laboratory and CSV for half of the variables, as

176 indicated by the LEG $\times$ LAB $\times$ CSV three-way interaction (Table 1, Supplementary Figs 2 and 3).

177 For the other half, significant two-way interactions between LEG $\times$ LAB and CSV $\times$ LAB were

178 found. The same significant interactions were found when analyzing the first (PC1) and second

179 (PC2) principal components from a principal component analysis (PCA) that included all 12

180 response variables; PC1 and PC2 together explained $45 \%$ of the variation (Table 1;

181 Supplementary Fig. 4ab). Taken together, these results suggest that the effect size or direction of

182 the net legume effect was significantly different (i.e. not reproducible) in some laboratories and

183 that the introduced CSV treatment affected reproducibility. In a complementary analysis

184 including the SETUP in the model (and accounting for the LAB effect as a random factor), we

185 found that the impact of the CSV treatment varied significantly with the SETUP $(\mathrm{CSV} \times \mathrm{SETUP}$

186 or LEG $\times C S V \times$ SETUP interactions; Supplementary Table 3), suggesting the reproducibility of

187 the results differed between glasshouses and growth chambers.

188 To answer the question of how many laboratories produced results that were statistically

189 indistinguishable from one another (i.e. reproduced the same finding), we used Tukey's post-hoc

190 Honest Significant Difference (HSD) test for the LAB effect on the first and second principal

191 components describing the net legume effect, which together explained $49 \%$ of the variation

192 (Supplementary Fig. 4cd). Out of the 14 laboratories, seven (PC1) and 11 (PC2) laboratories

193 were statistically indistinguishable in controls; this value increased in the treatments with

194 environmental or genotypic CSV for PC1 but not PC2 (Table 2). When we analyzed responses in 
195 growth chambers alone, five of eight laboratories were statistically indistinguishable in controls,

196 but this increased to six out of eight laboratories when we considered treatments with only

197 environmental CSV and seven of eight in treatments with genotypic CSV (GEN $\mathrm{GEN}_{\mathrm{A}}$ and

$\left.198 \mathrm{GEN}_{\mathrm{W}}+\mathrm{ENV}_{\mathrm{w}}\right)$. In glasshouses, introducing CSV did not affect the number of statistically

199 indistinguishable laboratories with respect to PC1 but decreased the number of statistically

200 indistinguishable laboratories with respect to PC2 (Table 2).

201 We also assessed the impact of the experimental treatments on the among- and within-

202 laboratory SD. Analysis of the among-laboratory SD of the net legume effect revealed a

203 significant CSV $\times$ SETUP interaction $\left(\mathrm{F}_{5,121}=7.38, \mathrm{P}<0.001\right)$ (Fig. 3a, b). This interaction

204 included significantly lower fitted coefficients (i.e., lower among-laboratory SD) in growth

205 chambers for $\operatorname{GEN}_{\mathrm{W}}\left(t_{5,121}=-3.37, P=0.001\right), \mathrm{GEN}_{\mathrm{A}}\left(t_{5,121}=-2.95, P=0.004\right)$ and

$206 \mathrm{ENV}_{\mathrm{W}}+\mathrm{GEN}_{\mathrm{W}}\left(t_{1,121}=-3.73, P<0.001\right)$ treatments relative to CTR (see full model output for

207 among-laboratory SD in Supplementary Note). For these three treatments, the among-laboratory

208 SD of the net legume effect was $18 \%$ lower with genotypic CSV than without it, indicating

209 increased reproducibility (Fig. 3a). The same analysis performed on within-laboratory SD of the

210 net legume effect only found a slight but significant increase of within-laboratory SD in the

$211 \mathrm{GEN}_{\mathrm{A}}$ treatment $\left(t_{5,121}=3.52, P<0.001\right)$ (see model output for within-laboratory SD in

212 Supplementary Note). We then tested whether there was a relationship between within- and

213 among-laboratory SD with a statistical model for among-laboratory SD as a function of within-

214 laboratory SD, SETUP, CSV and their interactions. We found a significant within-laboratory

$215 \operatorname{SD} \times$ SETUP $\times$ CSV three-way interaction $\left(F_{5,109}=2.4, P<0.040\right)$ affecting among-laboratory SD

216 (Supplementary Note). This interaction was the result of a more negative relationship between 
217 within- and among-laboratory SD in glasshouses relative to growth chambers, but with different 218 slopes for the different CSV treatments (Fig. 4).

219 Introducing CSV can increase within-laboratory variation, as indicated by the positive 220 coefficients fitted in some of the CSV treatments in the model output for within-laboratory SD 221 (see Supplementary Note). Thus, for the three CSV treatments that produced the most consistent 222 results $\left(\mathrm{GEN}_{\mathrm{W}}, \mathrm{GEN}_{\mathrm{A}}, \mathrm{ENV}_{\mathrm{W}}+\mathrm{GEN}_{\mathrm{W}}\right)$, we analyzed the statistical power of detecting the net 223 legume effect within individual laboratories. In growth chambers, adding genotypic CSV led to a 224 slight reduction in statistical power relative to CTR (57\% in CTR vs. $46 \%$ in the three treatments 225 containing genotypic variability) that could have been compensated for by using eleven instead 226 of six replicated microcosms per treatment. In glasshouses, owing to a higher effect size of 227 legume presence on the response variables, the statistical power for detecting the legume effect 228 in CTR was slightly higher (68\%) than in growth chambers, but was reduced to $51 \%$ on average 229 for the three treatments containing genotypic CSV, a decrease that could have been compensated 230 for by using 16 replicated microcosms instead of six.

231 Overall, our study shows that results produced by microcosm experiments can be strongly

232 biased by lab-specific factors. Based on the principal component explaining most of the variation 233 in the twelve response variables (PC1), only seven out of the 14 laboratories produced results 234 that can be considered reproducible (Table 2) with the current standardization procedures. This 235 result is in line with the only other comparable study ${ }^{12}$ (to the best of our knowledge) reporting 236 that out of ten laboratories, only four generated similar leaf growth phenotypes of Arabidopsis 237 thaliana (L). In addition to highlighting that approximately one in two ecological studies 238 performed in microcosms under controlled environments produce statistically different results, 239 our study provides supporting evidence for the hypothesis that introducing genotypic CSV can 
240 increase reproducibility of ecological studies ${ }^{9-11}$. However, the effectiveness of genotypic CSV

241 for enhancing reproducibility varied with the setup; it led to lower (-18\%) among-laboratory SD

242 in growth chambers only, with no benefit observed in glasshouses. Lower among-laboratory SD

243 in growth chambers implies that the microcosms containing genotypic CSV were less strongly

244 affected by unaccounted-for lab-specific environmental or biotic variables. Analyses performed

245 at the level of individual variables (Table 1) showed that introducing genotypic CSV affected the

246 among-laboratory SD in most, but not all variables. This suggests that the relationship between

247 genotypic CSV and reproducibility is probabilistic and results from the decreased likelihood that

248 microcosms containing CSV will respond to unaccounted for lab-specific environmental factors

249 in the same direction and with the same magnitude. The mechanism is likely to be analogous to

250 the stabilizing effect of biodiversity on ecosystem functions under changing environmental

251 conditions $^{23-26}$, but additional empirical evidence is needed to confirm this conjecture.

252 Introducing genotypic CSV increased reproducibility in growth chambers (with stringent

253 control of environmental conditions) but not in glasshouses (with more variable environmental

254 conditions). Higher among-laboratory SD in glasshouses may indicate the existence therein of

255 stronger laboratory-specific factors, and our deliberate use of different soils in the glasshouses

256 presumably contributed to this effect. However, the among-laboratory SD in glasshouses

257 decreased with increasing within-laboratory SD, irrespective of CSV, an effect that was less

258 clear in growth chambers (Fig. 4). This observation appears to be in line with the hypothesis put

259 forward by Richter et al. ${ }^{9}$, who proposed that increasing the variance within experiments can

260 reduce the among-laboratory variability of the mean effect sizes observed in each laboratory.

261 Yet, despite the negative correlation between within- and among-laboratory SD observed in

262 glasshouses, the among-laboratory SD remained higher in glasshouses than in growth chambers. 
263 Therefore, we consider that the hypothesized mechanistic link between CSV-induced higher

264 within-laboratory SD and increased reproducibility is poorly supported by our dataset.

265 Nevertheless, one possible explanation for the lack of effect on reproducibility in glasshouses is

266 that our CSV treatments did not introduce a sufficiently high level of within-laboratory

267 variability to buffer against laboratory-specific factors for all response variables; across the

268 twelve response variables, the average main effect (i.e., without the interaction terms) of the

269 CSV treatment contributed to a low percentage ( $2.6 \% \pm 1.6$ s.e.m.) of the total sum of squares

270 relative to the main effects of laboratory $(43.4 \% \pm 5.2$ s.e.m.) and legumes $(10.9 \% \pm 3.1$ s.e.m.).

271 A similar conjecture was put forward by the other two studies that explored the role of CSV for

272 reproducibility in animal behavior ${ }^{9}, 10$. At present we are unable to conclude that the introduction

273 of stronger sources of controlled within-laboratory variability can increase reproducibility in

274 glasshouses with more loosely controlled environmental conditions and different soils.

275 Our results indicate that genotypic CSV is more effective in increasing reproducibility than

276 environmental CSV, irrespective of whether the CSV was introduced within or among individual

277 replicates (i.e., microcosms). However, we cannot discount the possibility that we found this

278 result because our treatments with environmental CSV were less successful in increasing within-

279 microcosm variability. Additional experiments could test whether other types of environmental

280 CSV, such as soil nutrients, texture, or water availability, might be more effective at increasing

281 reproducibility.

282 We expected higher overall productivity (i.e., a net legume effect) in the grass-legume

283 mixtures and enhanced growth of $B$. distachyon because of the presence of the nitrogen $(\mathrm{N})$ -

284 fixing M. truncatula. However, these species were not selected because of their routine pairings

285 in agronomic or ecological experiments (they are rarely used that way), but rather because they 
286 are frequently present in controlled environment experiments looking at functional genomics.

287 Contrary to our expectation, and despite the generally lower ${ }^{15} \mathrm{~N}$ signature of $B$. distachyon in the 288 presence of $\mathrm{N}$-fixing $M$. truncatula (suggesting that some of the $\mathrm{N}$ fixed by M. truncatula was 289 taken up by the grass), the biomass of $B$. distachyon was lower in the microcosms containing $M$.

290 truncatula. Seed mass and shoot \% $\mathrm{N}$ data of B. distachyon was lower in mixtures

291 (Supplementary Fig. 1), suggesting that the two species competed for N. The lack of a significant

292 N fertilization effect of M. truncatula on B. distachyon could have resulted from the

293 asynchronous phenologies of the two species: the 8-10-week life cycle of $B$. distachyon may

294 have been too short to benefit from the N fixation by M. truncatula.

295 Because well-established meta-analytical approaches can account for variation caused by

296 local factors and still detect the general trends across different types of experimental setups, 297 environments, and populations, we should ask whether the additional effort required for 298 introducing CSV in experiments is worthwhile. Considering the current reproducibility crisis in 299 many fields of science ${ }^{27}$, we suggest that it is, for at least three reasons. First, some studies

300 become seminal without any attempts to reproduce them. Second, even if a seminal study that is 301 flawed due to laboratory-specific biases is later proven wrong, it usually takes significant time 302 and resources before its impact on the field abates. Third, the current rate of reproducibility is 303 estimated to be as low as one-third ${ }^{12-14}$, implying that most data entering any meta-analysis are

304 biased by unknown lab-specific factors. Addition of genotypic CSV may enhance the 305 reproducibility of individual experiments and eliminate potential biases in data used in meta306 analyses. Last, if each individual study is less affected by laboratory-specific unknown 307 environmental and biotic factors, then we would also need fewer studies to draw solid 308 conclusions about the generality of phenomena. Therefore, we argue that investing more in 
309 making individual studies more reproducible and generalizable will be beneficial in both the

310 short and long run. At the same time, adding CSV can reduce statistical power to detect

311 experimental effects, so some additional experimental replicates would be needed when using it.

312 Arguably, our use of statistical significance tests of effects sizes to determine reproducibility

313 might be viewed as overly restrictive and better suited to assessing reproducibility of parameter

314 estimates rather than assessing the generality of the hypothesis under test ${ }^{27}$. We used this

315 approach because no generally accepted alternative framework is available to assess how close

316 the multivariate results from multiple laboratories need to be to conclude that they reproduced

317 the same finding. It is worth noting that although the direction of the legume effect was the same

318 in the majority of laboratories, the differences among laboratories were very large (e.g., up to

319 two orders of magnitude for shoot biomass) and in $10 \%$ of the 168 laboratory $\times$ variable

320 combinations (14 laboratories $\times 12$ response variables) the direction of the legume effect differed

321 from the among-laboratory consensus (Fig. 2).

322 In conclusion, our study shows that the current standardization procedures used in ecological

323 microcosm experiments are inadequate in accounting for lab-specific environmental factors and

324 suggests that introducing controlled variability in experiments may buffer effects of lab-specific

325 factors. Although there are multiple causes for the reproducibility crisis $^{15,28,29}$, deliberately

326 including genetic variation in the studied organisms can be a simple solution for increasing the

327 reproducibility of ecological studies performed in controlled environments. However, as the

328 introduced genotypic variability only increased reproducibility in experimental setups with

329 tightly controlled environmental conditions (i.e., in growth chambers using identical soil), our

330 study indicates that the reproducibility of ecological experiments can be enhanced by a 
331 combination of rigorous standardization of environmental variables at the laboratory level as

332 well as controlled genotypic variability.

\section{References}

335 1. Cassey, P. \& Blackburn, T. Reproducibility and Repeatability in Ecology. Bioscience 56, 336 958-9 (2006).

337 2. Ellison, A. M. Repeatability and transparency in ecological research. Ecology 91, 2536$338 \quad 2539(2010)$

339 3. Lawton, J. H. The Ecotron facility at Silwood Park: the value of ' big bottle' experiments. $340 \quad$ Ecology 77, 665-669 (1996).

341 4. Benton, T. G., Solan, M., Travis, J. M. \& Sait, S. M. Microcosm experiments can inform 342 global ecological problems. Trends Ecol. Evol. 22, 516-521 (2007).

343 5. Drake, J. M. \& Kramer, A. M. Mechanistic analogy: how microcosms explain nature.

$344 \quad$ Theor. Ecol. 5, 433-444 (2012).

345 6. Fraser, L. H. \& Keddy, P. The role of experimental microcosms in ecological research.

346 Trends Ecol. Evol. 12, 478-481 (1997).

347 7. Srivastava, D. S. et al. Are natural microcosms useful model systems for ecology? Trends $348 \quad$ Ecol. Evol. 19, 379-384 (2004).

349 8. De Boeck, H. J. et al. Global change experiments: challenges and opportunities.

$350 \quad$ Bioscience (2015). doi:10.1093/biosci/biv099

351 9. Richter, S. H. et al. Effect of population heterogenization on the reproducibility of mouse 352 behavior: a multi-laboratory study. PLoS One 6, e16461 (2011).

353 10. Richter, S. H., Garner, J. P. \& Würbel, H. Environmental standardization: cure or cause of 
poor reproducibility in animal experiments? Nat. Methods 6, 257-261 (2009).

355 11. Richter, S. H., Garner, J. P., Auer, C., Kunert, J. \& Würbel, H. Systematic variation

12. Massonnet, C. et al. Probing the reproducibility of leaf growth and molecular phenotypes: a comparison of three Arabidopsis accessions cultivated in ten laboratories. Plant Physiol. 152, 2142-2157 (2010).

13. Begley, C. G. \& Ellis, M. L. Raise standards for preclinical cancer research. Nature 483, $531-533$ (2012).

14. Open Science Collaboration. Estimating the reproducibility of psychological science. Science (80-. ). 349, aac4716 (2015).

15. Parker, T. H. et al. Transparency in ecology and evolution: real problems, real solutions. Trends Ecol. Evol. 31, 711-719 (2016).

16. Moore, R. P. \& Robinson, W. D. Artificial bird nests, external validity, and bias in ecological field studies. Ecology 85, 1562-1567 (2004).

17. Temperton, V. M., Mwangi, P. N., Scherer-Lorenzen, M., Schmid, B. \& Buchmann, N. species in a biodiversity experiment. Oecologia 151, 190-205 (2007).

19. Sleugh, B., Moore, K. J., George, J. R. \& Brummer, E. C. Binary legume-grass mixtures improve forage yield, quality, and seasonal distribution. Agron. J. 92, 24-29 (2000). 
Methods Ecol. Evol. 4, 1070-1075 (2013).

378 21. Nyfeler, D., Huguenin-Elie, O., Suter, M., Frossard, E. \& Lüscher, A. Grass-legume 379 mixtures can yield more nitrogen than legume pure stands due to mutual stimulation of 380 nitrogen uptake from symbiotic and non-symbiotic sources. Agric. Ecosyst. Environ. 140, $155-163$ (2011).

382 22. Suter, M. et al. Nitrogen yield advantage from grass-legume mixtures is robust over a 383 wide range of legume proportions and environmental conditions. Glob. Chang. Biol. 21, $2424-2438$ (2015).

385 23. Loreau, M. \& de Mazancourt, C. Biodiversity and ecosystem stability: A synthesis of underlying mechanisms. Ecol. Lett. 16, 106-115 (2013).

24. Reusch, T. B., Ehlers, A., Hämmerli, A. \& Worm, B. Ecosystem recovery after climatic extremes enhanced by genotypic diversity. Proc. Natl. Acad. Sci. U. S. A. 102, 2826 (2005).

25. Hughes, A. R., Inouye, B. D., Johnson, M. T. J., Underwood, N. \& Vellend, M. Ecological consequences of genetic diversity. Ecol. Lett. 11, 609-623 (2008).

392 26. Prieto, I. et al. Complementary effects of species and genetic diversity on productivity and stability of sown grasslands. Nat. Plants 1, 1-5 (2015).

394 27. Wasserstein, R. L. \& Lazar, N. A. The ASA's statement on p-values: context, process, and 395 purpose. Am. Stat. 70, 129-133 (2016).

396 28. Baker, M. 1,500 scientists lift the lid on reproducibility. Nature 533, 452-454 (2016).

397 29. Nuzzo, R. How scientists fool themselves - and how they can stop. Nature 526, 182-185 (2015). 


\section{Acknowledgements}

401 This study benefited from the CNRS human and technical resources allocated to the

402 ECOTRONS Research Infrastructures and the state allocation 'Investissement d'Avenir' ANR-

403 11-INBS-0001 and from financial support by the ExpeER (grant no. 262060) consortium funded

404 under the EU-FP7 research program (FP2007-2013). Brachypodium seeds were kindly provided

405 by Richard Sibout (Observatoire du Végétal, Institut Jean-Pierre Bourgin, F-78026 Versailles

406 Cedex France) and Medicago seeds were supplied by Jean-Marie Prosperi (INRA Biological

407 Resource Centre, F-34060 Montpellier Cedex 1, France). We further thank Jean Varale, Gesa

408 Hoffmann, Paul Werthenbach, Oliver Ravel, Clement Piel and Damien Landais, David

409 Degueldre, Thierry Mathieu, Pierrick Aury, Nicolas Barthès, Bruno Buatois, Raphaëlle Leclerc

410 for assistance duing the study. For additional acknowledgements see Supplementary Information.

\section{Author contributions}

412 A.M. and J.R. designed the study with input from M.B, S.B and J-C.L. Substantial methodological

413 contributions were provided by M.B., S.S., T.G., L.R. and M.S-L. Conceptual feedback on an early

414 version was provided by G.F., N.E., J.R. and A.M.E. Data were analysed by A.M. with input from

415 A.M.E. A.M. wrote the manuscript with input from all co-authors. All co-authors were involved

416 in carrying out the experiments and/or analyses.

\section{Author Information}

418 The authors declare no conflict of interest. Correspondence and request for materials should be 419 addressed to Alexandru Milcu (alex.milcu@cnrs.fr). 


\section{METHODS}

423 All laboratories tried to the best of their abilities to carry out an identical experimental protocol.

424 Whereas not all laboratories managed to recreate precisely all details of the experimental

425 protocol, we considered this to be a realistic scenario under which ecological experiments using

426 microcosms are performed in glasshouses and growth chambers.

\section{Germination}

428 The seeds from the three genotypes of Brachypodium distachyon (Bd21, Bd21-3 and Bd3-1) and 429 Medicago truncatula (L000738, L000530 and L000174) were first sterilized by soaking 100

430 seeds in $100 \mathrm{~mL}$ of a sodium hypochlorite solution with $2.6 \%$ active chlorine, and stirred for 15

431 min using a magnet. Thereafter, the seeds were rinsed 3 times in $250 \mathrm{~mL}$ of sterile water for 10-

43220 seconds under shaking. Sterilized seeds were germinated in trays $(10 \mathrm{~cm}$ deep $)$ filled with

433 vermiculite. The trays were kept at $4^{\circ} \mathrm{C}$ in the dark for three days before being moved to light

434 conditions (300 $\left.\mu \mathrm{mol} \mathrm{m} \mathrm{m}^{-2} \mathrm{~s}^{-1} \mathrm{PAR}\right)$ and $20 / 16^{\circ} \mathrm{C}$ and $60 / 70 \%$ air $\mathrm{RH}$ for day- and night-time,

435 respectively. When the seedlings of both species reached $1 \mathrm{~cm}$ in height above the vermiculite,

436 they were transplanted into the microcosms.

\section{Preparation of microcosms}

438 All laboratories used identical containers (2-liter volume, 14.8-cm diameter, 17.4-cm height).

439 Sand patches were created using custom-made identical "patch makers" consisting of six rigid

440 PVC tubes $(2.5 \mathrm{~cm}$ in diameter and $25 \mathrm{~cm}$ long), arranged in a circular pattern with an outer

441 diameter of $10 \mathrm{~cm}$. A textile mesh was placed at the bottom of the containers to prevent the

442 spilling of soil through drainage holes. Filling of microcosms containing sand patches started

443 with the insertion of the empty tubes into the containers. Thereafter, in growth chambers, 2000-g

444 dry-weight of soil, subtracting the weight of the sand patches, was added into the containers and 
445 around the "patch maker" tubes. Because different soils were used in the glasshouses, the dry

446 weight of the soil differed depending on the soil density and was first estimated individually in

447 each laboratory as the amount of soil needed to fill the pots up to $2 \mathrm{~cm}$ from the top. After the

448 soil was added to the containers, the tubes were filled with a mixture of $10 \%$ soil and $90 \%$ sand.

449 When the microcosms did not contain sand patches, the amount of sand otherwise contained in

450 the six patches was homogenized with the soil. During the filling of the microcosms, a common

451 substrate for measuring litter decomposition was inserted at the center of the microcosm at $8 \mathrm{~cm}$

452 depth. For simplicity as well as for its fast decomposition rate, we used a single batch of

453 commercially available tetrahedron-shaped synthetic tea bags (mesh size of $0.25 \mathrm{~mm}$ ) containing

$4542 \mathrm{~g}$ of green tea (Lipton, Unilever), as proposed by the "tea bag index" method ${ }^{20}$. Once filled, the

455 microcosms were watered until water could be seen pouring out of the pot. The seedlings were

456 then manually transplanted to predetermined positions (Fig. 1), depending on the genotype and

457 treatment. Each laboratory established two blocks of 36 microcosms each, resulting in a total of

45872 microcosms per laboratory, with blocks representing two distinct chambers in growth

459 chamber setups or two distinct growth benches in the same glasshouse.

460 Soils

461 All laboratories using growth chamber setups used the same soil, whereas the laboratories using 462 glasshouses used different soils (see Supplementary Table 1 for the physicochemical properties 463 of the soils). The soil used in growth chambers was classified as a nutrient-poor cambisol and 464 was collected from the top layer $(0-20 \mathrm{~cm})$ of a natural meadow at the Centre de Recherche en 465 Ecologie Expérimentale et Prédictive-CEREEP (Saint-Pierre-Lès-Nemours, France). Soils used 466 in glasshouses originated from different locations. The soil used by laboratory L2 was a fluvisol 467 collected from the top layer $(0-40 \mathrm{~cm})$ of a quarry site near Avignon, in the Rhône valley, 
468 Southern France. The soil used by laboratory L4 was collected from near the La Cage field 469 experimental system (Versailles, France) and was classified as a luvisol. The soil used by labs

470 L11 and L12 was collected from the top layer $(0-20 \mathrm{~cm})$ within the haugh of the river Dreisam in

471 the East of Freiburg, Germany. This soil was classified as an umbric gleysol with high organic

472 carbon content. The soil from laboratory L14 was classified as a eutric fluvisol and was collected

473 on the field site of the Jena Experiment, Germany. Prior to the establishment of microcosms, all

474 soils were air-dried at room temperature for several weeks and sieved with a 2-mm mesh sieve.

475 A common inoculum was provided to all laboratories to assure that rhizobia specific to $M$.

476 truncatula were present in all soils.

\section{Abiotic environmental conditions}

478 The set points for environmental conditions were $16 \mathrm{~h} \mathrm{light} \mathrm{(at} 300 \mu \mathrm{mol} \mathrm{m} \mathrm{m}^{-2} \mathrm{~s}^{-1} \mathrm{PAR}$ ) and $8 \mathrm{~h}$ 479 dark, $20 / 16^{\circ} \mathrm{C}, 60 / 70 \%$ air $\mathrm{RH}$ for day- and night-time, respectively. Different soils (for 480 glasshouses) and treatments with sand patches likely affected water drainage and 481 evapotranspiration. The watering protocol was thus based on dry weight relative to weight at full 482 water holding capacity (WHC). The WHC was estimated based on the weight difference between 483 the dry weight of the containers and the wet weight of the containers $24 \mathrm{~h}$ after abundant 484 watering (until water was flowing out of the drainage holes in the bottom of each container). Soil 485 moisture was maintained between 60 and $80 \%$ of WHC (i.e. the containers were watered when 486 the soil water dropped below $60 \%$ of WHC and water added to reach $80 \%$ of WHC) during the 487 first 3 weeks after seedling transplantation and between 50 and $70 \%$ of WHC for the rest of the 488 experiment. Microcosms were watered twice a week with estimated WHC values from two 489 microcosms per treatment. To ensure that the patch/heterogeneity treatments did not become a 490 water availability treatment, all containers were weighed and brought to 70 or $80 \%$ of WHC 
491 every two weeks. This operation was synchronized with within-block randomization. All 14

492 experiments were performed between October 2014 and March 2015.

493 Sampling and analytical procedures

494 After 80 days, all plants were harvested. Plant shoots were cut at the soil surface, separated by 495 species, and dried at $60^{\circ} \mathrm{C}$ for three days. Roots and any remaining litter in the tea bags were 496 washed out of the soil using a $1-\mathrm{mm}$ mesh sieve and dried at $60^{\circ} \mathrm{C}$ for three days. Microcosm 497 evapotranspiration rate was measured before the harvesting as the difference in weight changes 498 from $70 \%$ of WHC after $48 \mathrm{~h}$. Shoot $\mathrm{C} \%, \mathrm{~N} \%, \delta^{13} \mathrm{C}$, and $\delta^{15} \mathrm{~N}$ were measured on pooled shoot 499 biomass (including seeds) of B. distachyon and analyzed at the Göttingen Centre for Isotope 500 Research and Analysis using a coupled system consisting of an elemental analyzer (NA 1500, 501 Carlo Erba, Milan, Italy) and a gas isotope mass spectrometer (MAT 251, Finnigan, Thermo 502 Electron Corporation, Waltham, Massachusetts, USA).

\section{Data analysis and statistics}

$504 \quad$ All analyses were done using R version 3.2.4 ${ }^{29}$. Prior to data analyses, each laboratory was 505 screened individually for outliers. Values that were lower or higher than $1.5 \times$ IQR (interquartile 506 range $)^{30}$ within each laboratory, and representing less than $1.7 \%$ of the whole dataset, were 507 considered to be outliers due to measurement errors or typos. These values were removed and 508 subsequently treated as missing values. We then assessed whether the impact of the presence of

509 legume (LEG) varied with laboratory (LAB) and the treatment of controlled systematic

510 variability (CSV). This was tested individually for each response variable (Table 1) with a 511 mixed-effects model using the "nlme" package ${ }^{31}$. Following the guidelines suggested by Zuur et 512 al. $(2009)^{32}$, we first identified the most appropriate random structure using a restricted

513 maximum likelihood (REML) approach and selected the random structure with the lowest 
514 Akaike information criterion (AIC). For this model, CSV and LAB were included as fix factors,

515 experimental block as a random factor, and a "varIdent" weighting function to correct for

516 heteroscedasticity resulting from more heteroscedastic data at the LAB and LEG level (R syntax:

517 "model $=$ lme (response variable $\sim \mathrm{LEG}^{*} \mathrm{CSV} * \mathrm{LAB}$, random= 1|block, weights=varIdent (form

$\left.518=\sim 1 \mid \mathrm{LAB}^{*} \mathrm{LEG}\right)^{\prime \prime}$ ) (Table 2). As the LAB and SETUP experimental factors were not fully

519 crossed (i.e. laboratories performed the experiment only in one type of setup), the two

520 experimental variables could not be included simultaneously as fixed effects. Therefore, to test

521 for the SETUP effect, we used an additional complementary model including CSV and SETUP

522 as fix effects and laboratory as a random factor (R syntax: "model= lme (response variable

523 LEG*CSV*SETUP, random= 1|LAB/block, weights=varIdent (form = 1|LAB*LEG)")

524 (Supplementary Table 3). To test whether the results were affected by the collinearity among the 525 response variables, the two models also were run on the first (PC1) and second (PC2) principal 526 components the 12 response variables (Fig. 4ab). PCs were estimated using the "FactoMineR" 527 package ${ }^{33}$, with missing values replaced using a regularized iterative multiple correspondence 528 analysis $^{34}$ in the "missMDA" package ${ }^{35}$. The same methodology was used to compute a second 529 PCA derived from the net legume effect on the 12 response variables (Supplementary Fig. 4cd).

530 To assess how many laboratories produced results that were statistically indistinguishable from 531 one another, we applied Tukey's post-hoc HSD test in the "multcomp" package to lab-specific 532 estimates of PC1 and PC2 (Table 2).

533 To assess how the CSV treatments affected the among- and within-laboratory variability, 534 we used the standard deviation (SD) instead of the coefficient of variation, because the net 535 legume effect contained both positive and negative values. To calculate among- and within536 laboratory SDs, we centered and scaled the raw values using the $z$-score normalization $[z$-scored 
537 variable $=($ raw value-mean $) / \mathrm{SD}]$ individually for each of the 12 response variables. Among-

538 laboratory SD was computed from the mean of the laboratory $z$-scores for each response

539 variable, CSV, and SETUP treatments $(\mathrm{n}=144 ; 6 \mathrm{CSV}$ levels $\times 2$ SETUP levels $\times 12$ response

540 variables). Within-laboratory SDs were computed from the values measured in the six replicated

541 microcosms for each CSV and SETUP treatment combination, individually for each response

542 variable, resulting in a dataset with the same structure as for among-laboratory SDs ( $\mathrm{n}=144 ; 6$

543 CSV levels $\times 2$ SETUP levels $\times 12$ response variables). Some of the 12 response variables were

544 intrinsically correlated, but most had correlation coefficients < 0.5 (Supplementary Fig. 5) and

545 were therefore treated as independent variables. To analyze and visualize the relationships

546 between the SDs calculated from variables with different units, before the calculation of the

547 among- and within-laboratory SD, the raw values of the 12 response variables were centered and 548 scaled.

549 The impact of experimental treatments on among- and within-laboratory SD was analyzed 550 using mixed-effect models, following the same procedure described for the individual response 551 variables. The model with the lowest AIC included a random slope for the SETUP within each 552 response variable as well as a "varIdent" weighting function to correct for heteroscedasticity at 553 the variable level (R syntax: "model= lme (SD CSV*SETUP, random= SETUP|variable, 554 weights $=$ varIdent (form $=\sim 1 \mid$ variable)) (see also Supplementary Notes). The relationship 555 between within- and among-laboratory SD also was tested with a model with similar random 556 structure but with among-laboratory SD as a dependent variable and within-laboratory SD, CSV, 557 and SETUP as predictors.

558 Because the treatments containing genotypic CSV increased reproducibility in growth 559 chambers, but slightly increased within-laboratory SD, we also examined the effect of adding 
$560 \mathrm{CSV}$ on the statistical power for detecting the net legume effect in each individual laboratory.

561 This analysis was done with the "power.anova.test" function in the "base" package. We

562 computed the statistical power of detecting a significant net legume effect (if one had used a one-

563 way ANOVA for the legume treatment) for CTR, GEN ${ }_{W}, G E N_{A}$ and $E N V_{W}+G E N_{W}$ treatments

564 for each laboratory and response variable. This allowed us to calculate the average statistical

565 power for the aforementioned treatments and how many additional replicates would have been

566 needed to achieve the same statistical power as we had in the CTR.

567 The data that support the findings of this study are publicly available at

568 https://doi.pangaea.de/10.1594/PANGAEA.880980

569 Additional References for methods

570 30. R Development Core Team. R: a language and environment for statistical computing. R

$571 \quad$ Foundation for Statistical Computing, Vienna, Austria. (2017).

572 31. Tukey, J. W. Exploratory Data Analysis. (1977).

573 32. Pinheiro, J., Bates, D., DebRoy, S. \& Sarkar, D. NLME: Linear and nonlinear mixed$574 \quad$ effects models. $R$ Packag. version 3.1-122, http//CRAN.R-project.org/package=nlme 1$575 \quad 336(2016)$.

576 33. Zuur, A. F., Ieno, E. N., Walker, N., Saveliev, A. a \& Smith, G. M. Mixed-effects Models 577 and Extension in Ecology with R. (2009).

578 34. Lê, S., Josse, J. \& Husson, F. FactoMineR: An R package for multivariate analysis. $J$. $579 \quad$ Stat. Softw. 25, 1-18 (2008).

580 35. Josse, J., Chavent, M., Liquet, B. \& Husson, F. Handling missing values with regularized 581 iterative multiple correspondance analysis. J. Classif. 29, 91-116 (2010). 
Milcu et al. 2017

582 36. Josse, J. \& Husson, F. missMDA : A package for handling missing values in multivariate 583 data analysis. J. Stat. Softw. 70, 1-31 (2016). 
Table 1 | Impact of experimental treatments on response variables. Mixed-effects model outputs summarizing the F- and P-values (as asterisks) for the impacts of the presence of legumes (LEG), controlled systematic variability (CSV) and laboratory (LAB) on the 12 response variables. We also present the impact of experimental treatments on the first and second principal components (PC1 and PC2) of all 12 response variables. The response variables we measured are a typical ensemble of variables measured in plant-soil microcosm experiments ( $\mathrm{BM}=$ biomass $). \dagger$ symbol indicates response variables measured for the grass $B$. distachyon only, whereas the rest of the variables were measured at the microcosm level, i.e. including the contribution of both the legume and the grass species. Asterisks indicate the significance levels (*** for $P<0.001$; ** for $P<0.01$; * for $P<0.05$; + for $P<0.1$; ns for $P>0.1$ ). DF $=$ numerator degrees of freedom.

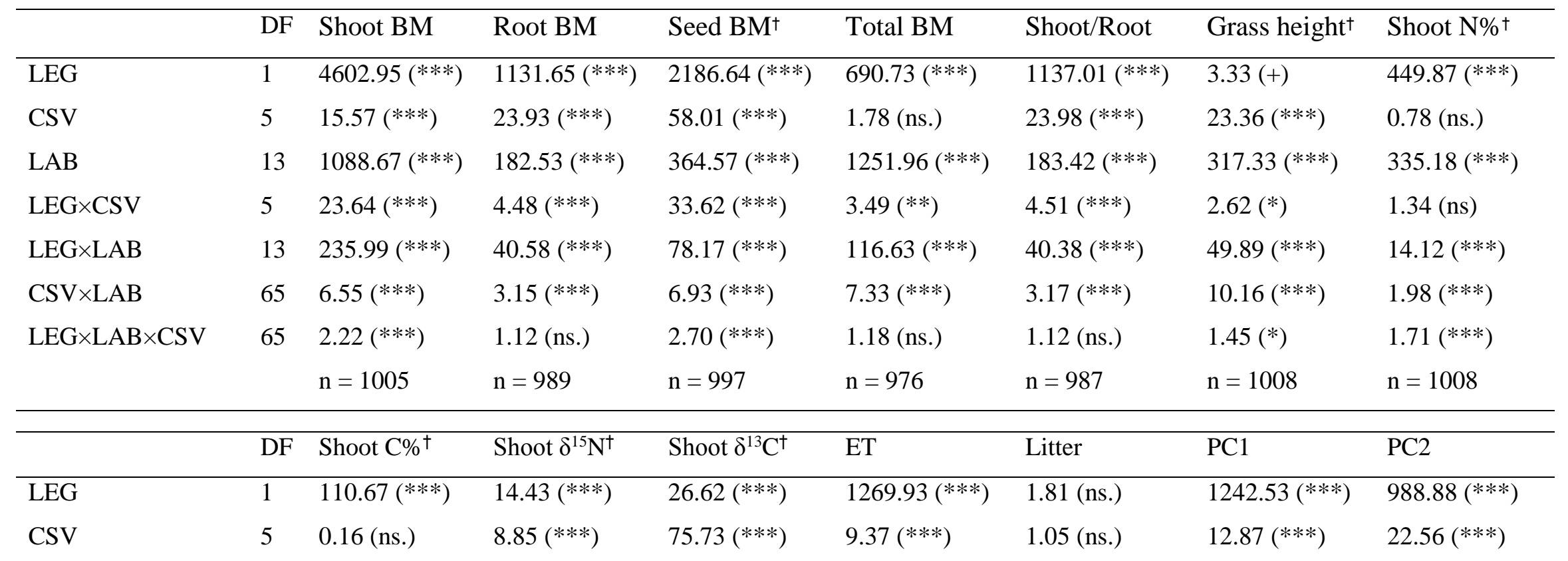




\begin{tabular}{|c|c|c|c|c|c|c|c|c|}
\hline LAB & 13 & $174.50(* * *)$ & $258.30(* * *)$ & $888.42(* * *)$ & $748.66(* * *)$ & $117.34(* * *)$ & $920.65(* * *)$ & $513.83(* * *)$ \\
\hline LEG×CSV & 5 & $2.55(*)$ & $6.48(* * *)$ & $5.15(* * *)$ & 1.24 (ns.) & 1.77 (ns.) & $7.08(* * *)$ & $11.79(* * *)$ \\
\hline LEG $\times$ LAB & 13 & $11.90(* * *)$ & $16.78(* * *)$ & $2.52(* *)$ & $172.74(* * *)$ & $2.05(*)$ & $118.12(* * *)$ & $28.22(* * *)$ \\
\hline \multirow[t]{2}{*}{$\mathrm{LEG} \times \mathrm{LAB} \times \mathrm{CSV}$} & 65 & $1.33(*)$ & $1.84(* * *)$ & 1.23 (ns.) & $1.53(* *)$ & 1.17 (ns.) & 0.93 (ns.) & $1.65(* *)$ \\
\hline & & $\mathrm{n}=1008$ & $\mathrm{n}=963$ & $\mathrm{n}=973$ & $\mathrm{n}=1002$ & $\mathrm{n}=974$ & $\mathrm{n}=1008$ & $\mathrm{n}=1008$ \\
\hline
\end{tabular}


595 Table 2 | Impact of experimental treatments on the number of laboratories that reproduced the

596 same finding. Numbers represent the total number of statistically indistinguishable laboratories based 597 on a Tukey's post-hoc Honest Significant Difference test of the first (PC1) and second (PC2) principal 598 components of the net legume effect of the 12 response variables (see Supplementary Fig. 4cd for the 599 PCA results). For a detailed description of experimental treatments and abbreviations, see Fig. 1.

600

\begin{tabular}{lcccccc}
\hline Source & $\begin{array}{c}\text { All laboratories } \\
(\mathrm{n}=14)\end{array}$ & \multicolumn{2}{c}{$\begin{array}{c}\text { Glasshouses } \\
(\mathrm{n}=6)\end{array}$} & \multicolumn{2}{c}{$\begin{array}{c}\text { Growth chambers } \\
(\mathrm{n}=8)\end{array}$} \\
\hline CTR & PC1 & PC2 & PC1 & PC2 & PC1 & PC2 \\
ENV $_{\mathrm{W}}$ & 7 & 11 & 3 & 5 & 5 & 5 \\
ENV $_{\mathrm{A}}$ & 10 & 9 & 3 & 3 & 6 & 6 \\
$\mathrm{GEN}_{\mathrm{W}}$ & 8 & 8 & 3 & 4 & 6 & 6 \\
$\mathrm{GEN}_{\mathrm{A}}$ & 8 & 10 & 3 & 3 & 6 & 7 \\
ENV $_{\mathrm{W}}+\mathrm{GEN}_{\mathrm{W}}$ & 11 & 10 & 3 & 3 & 7 & 8 \\
\hline
\end{tabular}

601

602 


\section{Figure legends}

604

Fig. 1 | Experimental design of one block. Grass monocultures of Brachypodium distachyon (green shades) and grass-legume mixtures with the legume Medicago trunculata (orange-brown shades) were established in 14 laboratories; shades of green and orange-brown represent three distinct genotypes of B. distachyon (Bd21, Bd21-3 and Bd3-1) and M. truncatula (L000738, L000530 and L000174). Plants were established in a substrate with equal proportions of sand (black spots) and soil (white), with the sand being either mixed with the soil or concentrated in sand patches to induce environmental controlled systematic variability (CSV). Combinations of three distinct genotypes were used to establish genotypic CSV. Alongside a control (CTR) with no CSV and containing one genotype (L000738 and/or Bd21) in a homogenized substrate (soil-sand mixture), five different types of environmental or genotypic CSV were used as treatments: 1) within-microcosm environmental CSV $\left(E N V_{W}\right)$ achieved by spatially varying soil resource distribution through the introduction of six sand patches into the soil; 2) among-microcosm environmental CSV $\left(\mathrm{ENV}_{\mathrm{A}}\right)$, which varied the number of sand patches (none, three or six) among replicate microcosms; 3) within-microcosm genotypic CSV $\left(\mathrm{GEN}_{\mathrm{W}}\right)$ that used three distinct genotypes per species planted in homogenized soil in each microcosm; 4) among-microcosm genotypic $\mathrm{CSV}\left(\mathrm{GEN}_{\mathrm{A}}\right)$ that varied the number of genotypes (one, two or three) planted in homogenized soil among replicate microcosms; and 5) both genotypic and environmental CSV $\left(\mathrm{GEN}_{\mathrm{W}}+\mathrm{ENV}_{\mathrm{W}}\right)$ within microcosms that used six sand patches and three plant genotypes per species in each microcosm. The " $\times 3$ " indicates that the same genotypic and sand composition was repeated in three microcosms per block. The spatial arrangement of the microcosms in each block was re-randomized every two weeks. The blocks represent two distinct chambers in growth chamber setups, whereas in glasshouse setups the blocks represent two distinct growth benches in the same glasshouse. 


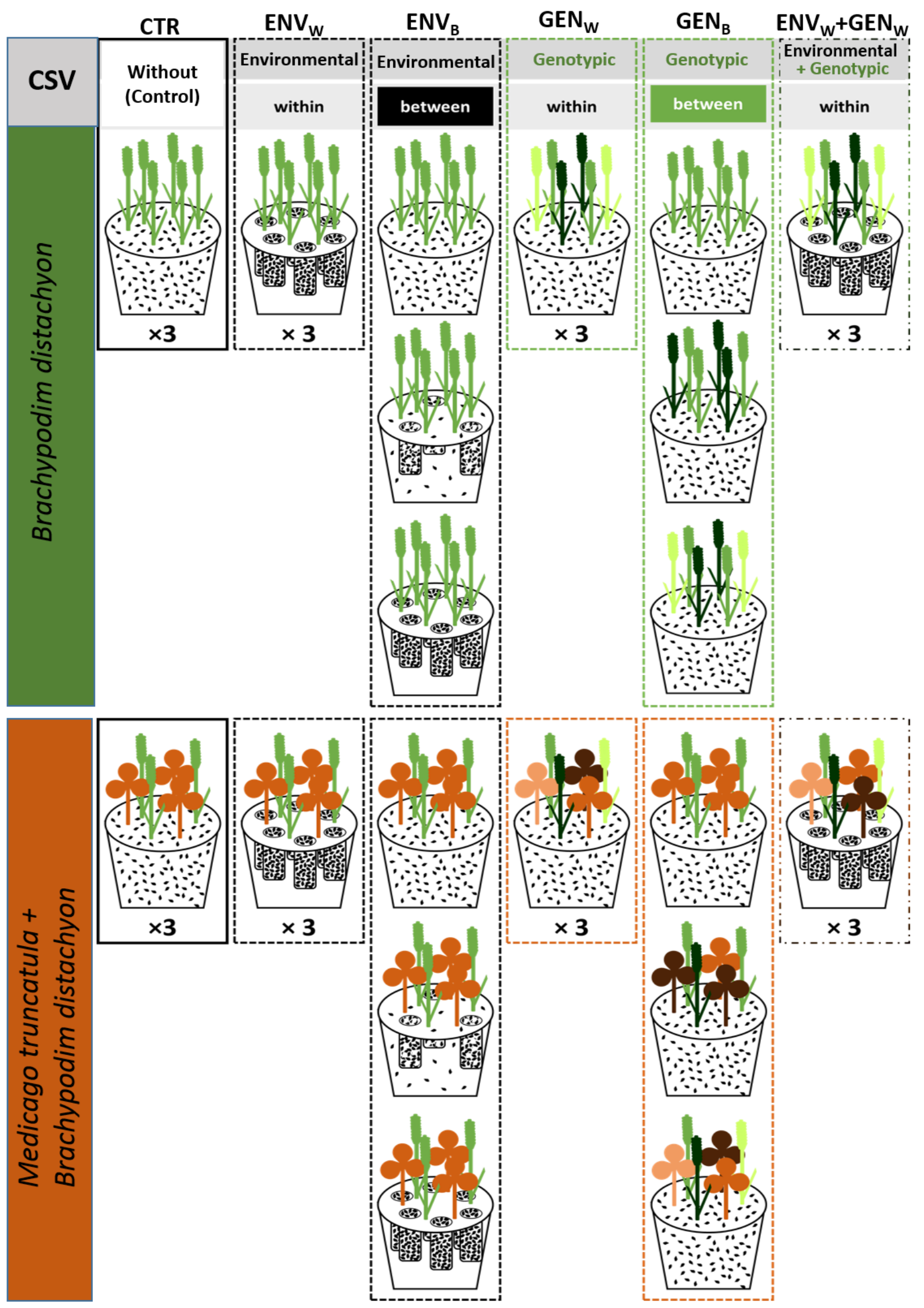


630 Fig. 2 | Net legume effect for the 12 response variables in 14 laboratories as affected by

631 laboratory and SETUP (growth chamber vs. glasshouse) treatment. The grey and blue bars

632 represent laboratories that used growth chamber and glasshouse set-ups, respectively. Bars show

633 means by laboratory obtained by averaging over all CSV treatments, with error bars indicating \pm 1

634 s.e.m. ( $\mathrm{n}=72$ microcosms per laboratory). 

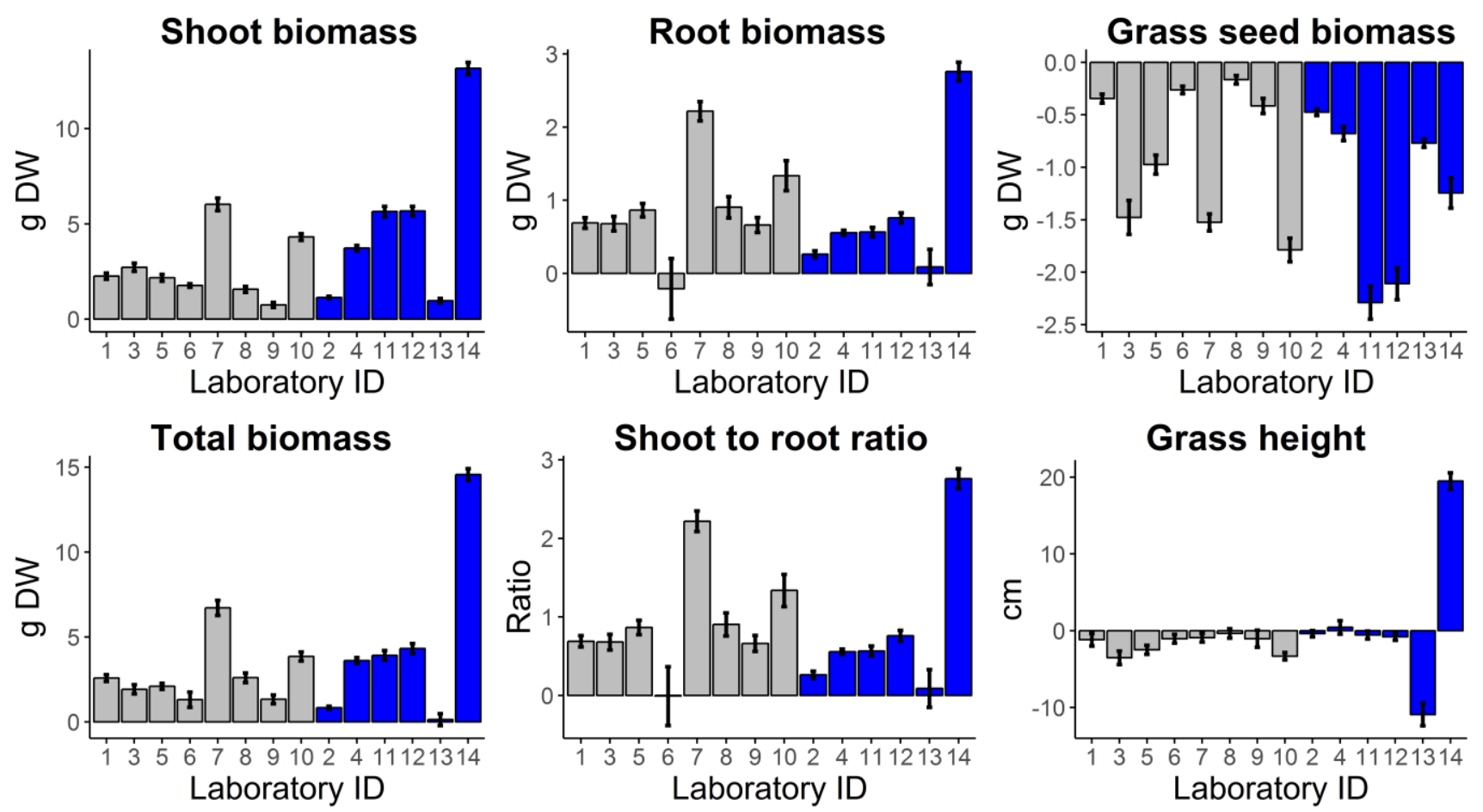

Grass foliar N

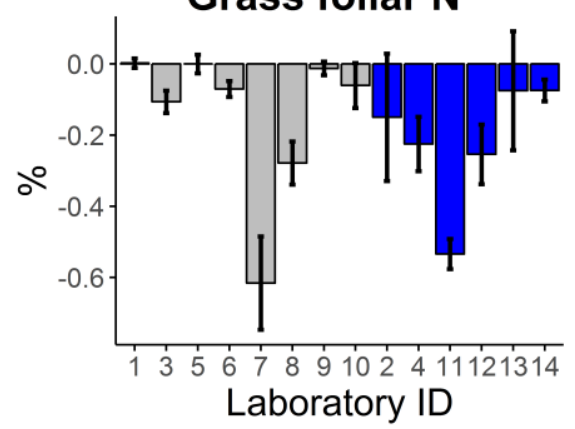

Grass foliar C
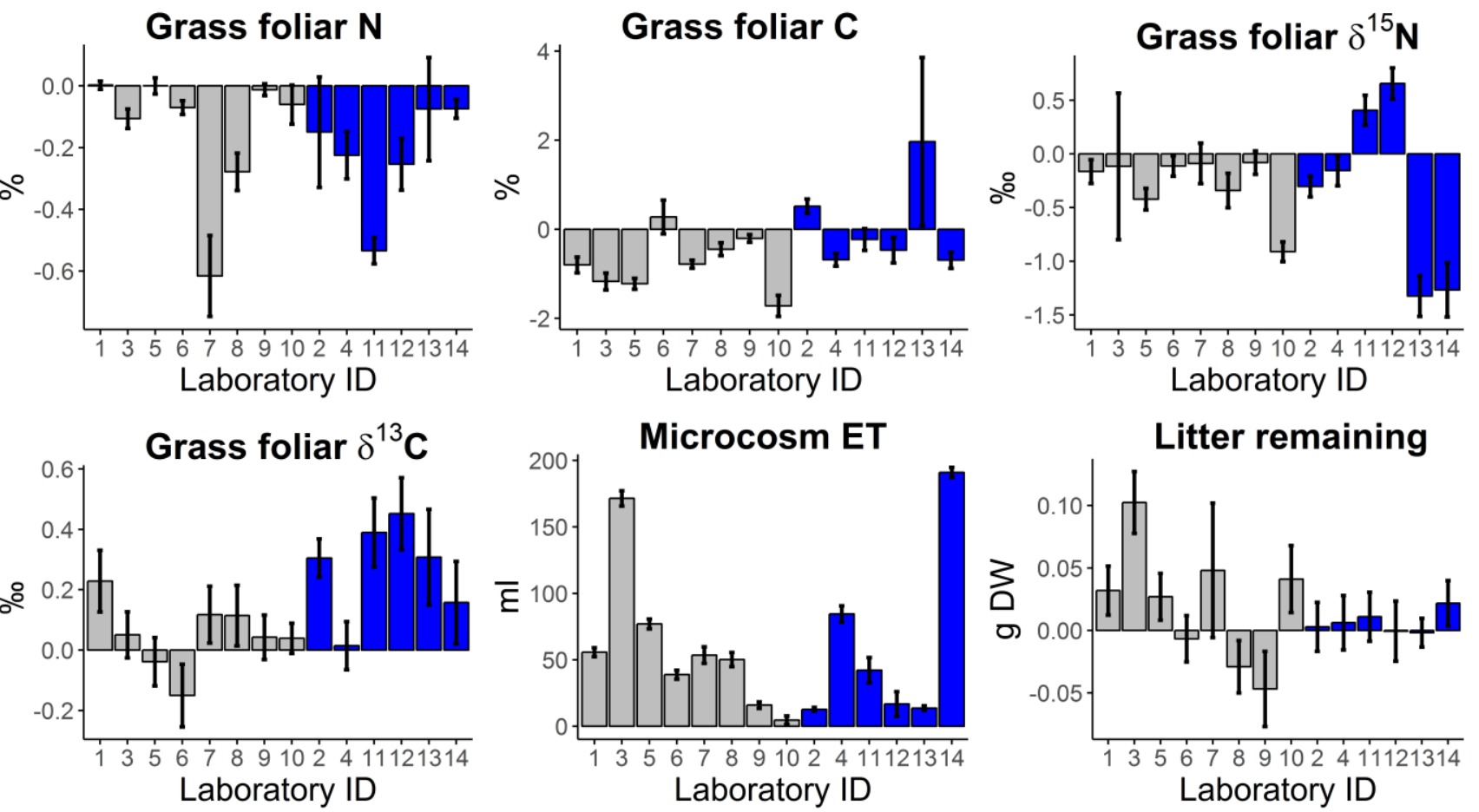

636 
639 Fig. 3 | Among- and within-laboratory standard deviation (SD) of the net legume effect as

640 affected by experimental treatments. Among-laboratory SD as affected by CSV and SETUP (a) and

641 SETUP only (b). Within-laboratory SD as affected by CSV and SETUP (c) and SETUP only (d).

642 Lower among-laboratory SD indicates enhanced reproducibility. Solid-filled bars and striped bars

643 represent glasshouse $(\mathrm{n}=6)$ and growth chamber setups $(\mathrm{n}=8)$, respectively. Asterisks represent $P$ -

644 values (*** for $P<0.001$, ** for $P<0.01$, * for $P<0.05$ ) indicating significantly different fitted

645 coefficients according to the mixed-effects models (see Supplementary Notes for full model outputs);

646 in (c) the star indicates the significant difference between $\mathrm{GEN}_{\mathrm{A}}$ and CTR, irrespective of the type of

647 SETUP. For a detailed description of experimental treatments and abbreviations see Fig. 1. 
$\square \mathrm{CTR} \square \mathrm{ENV}_{\mathrm{w}} \square \mathrm{ENV}_{\mathrm{A}} \square \mathrm{GEN}_{\mathrm{w}} \square \mathrm{GEN}_{\mathrm{A}} \square \mathrm{GEN}_{\mathrm{w}}+\mathrm{ENV}_{\mathrm{w}}$
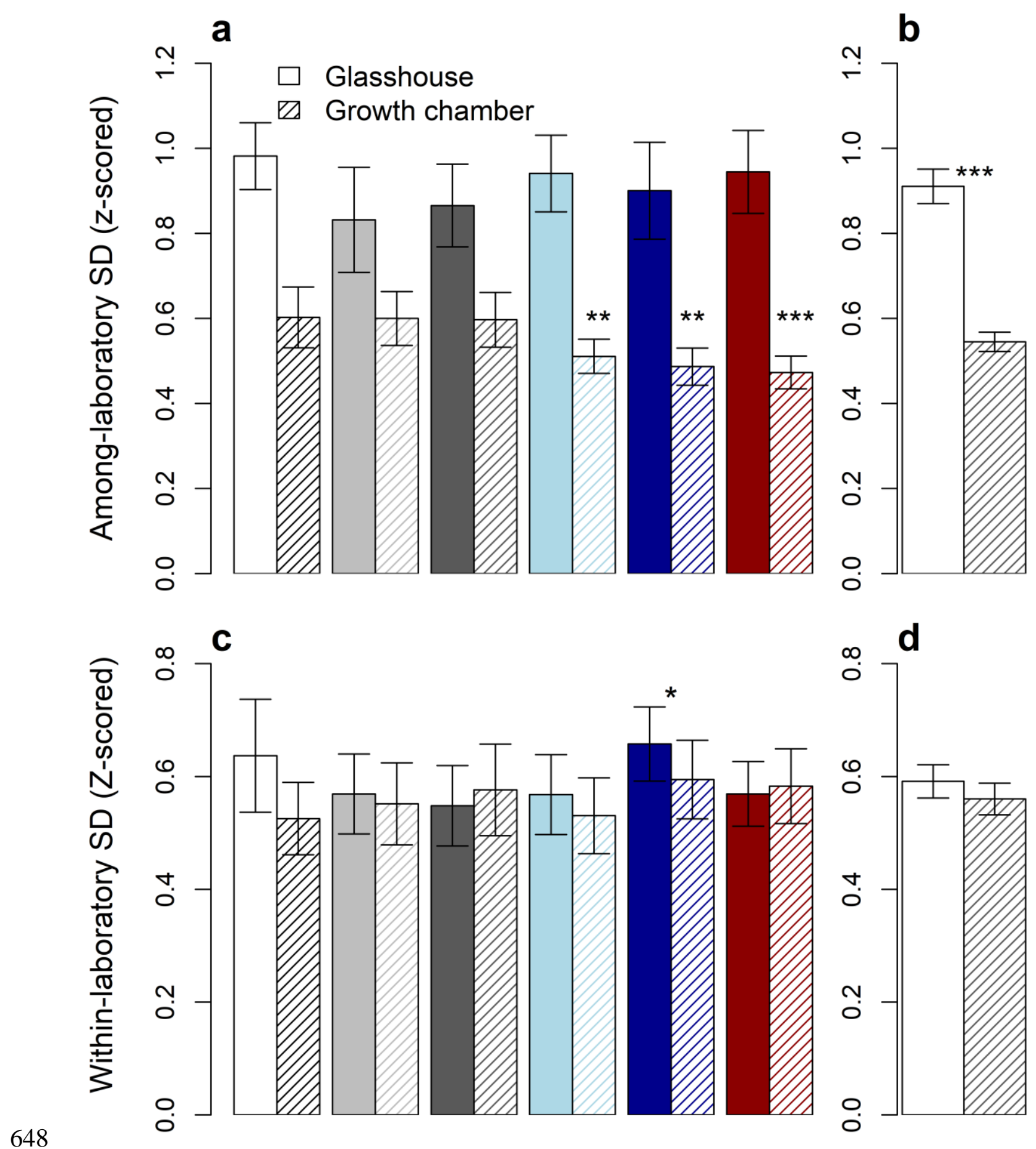

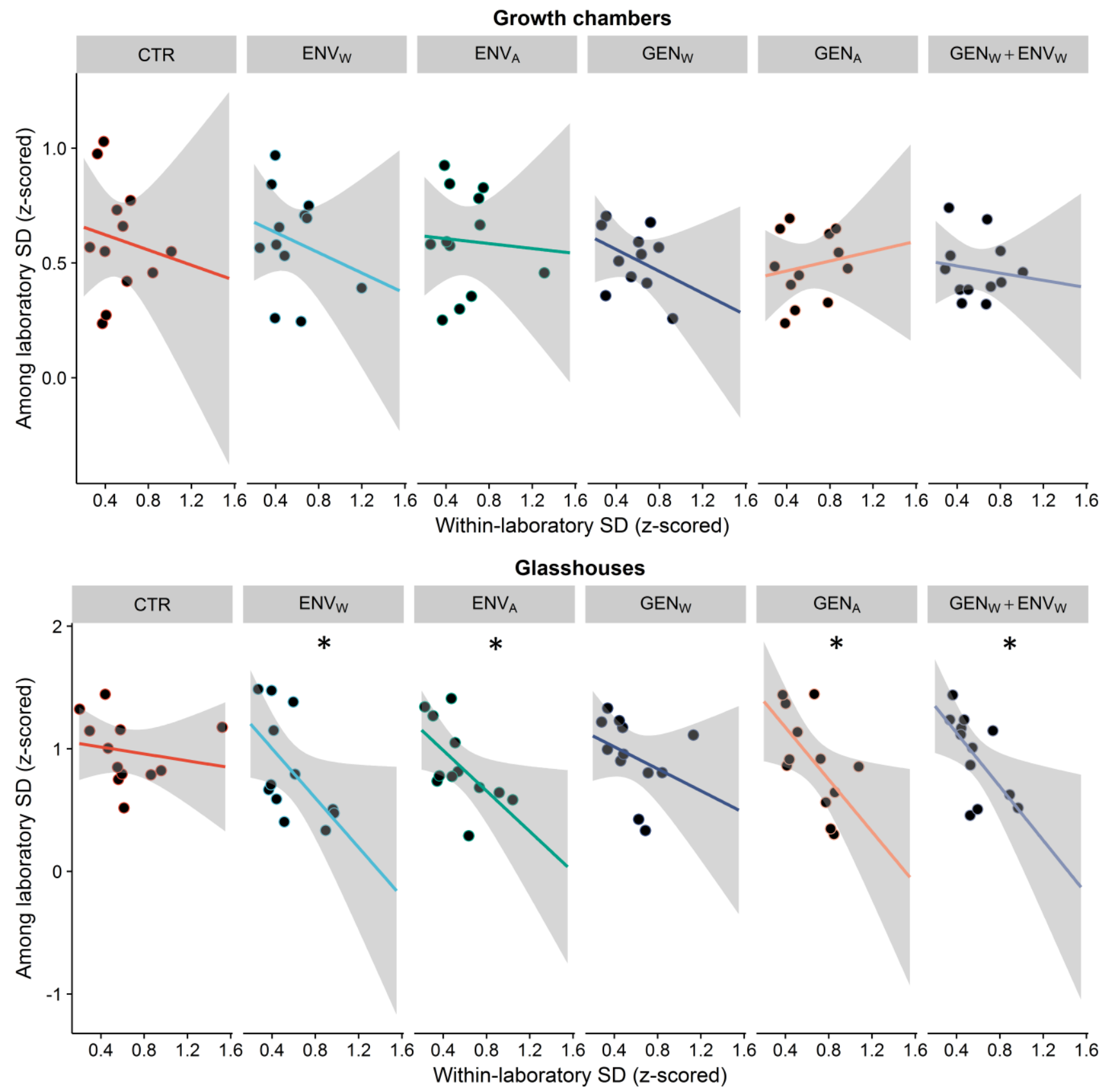

651 Fig. 4 | Relationship between within-laboratory SD and among-laboratory SD of the net legume

652 effect as affected by experimental treatments. The figure illustrates the significant within-laboratory

$653 \mathrm{SD} \times \mathrm{SETUP} \times \mathrm{CSV}$ three-way interaction $\left(\mathrm{F}_{5,109}=2.4, \mathrm{P}<0.040\right)$ affecting among-laboratory SD

654 (Supplementary Note). This interaction is the result of a more negative relationship between within-

655 and among-laboratory SD in glasshouses relative to growth chambers, but with different slopes for the

656 different CSV treatments. Points represent the 12 response variables. Asterisks represent $P$ values < 
Milcu et al. 2016

6570.05 for the individual linear regressions. Note the different scale for the y-axis between growth 658 chambers and glasshouses. For a detailed description of experimental treatments and abbreviations see 